\title{
POLARIZATION PLANE ROTATION EFFECTS ON SAR POLARIMETRIC ATTRIBUTES
}

\author{
Sidnei J. S. Sant'Anna ${ }^{(1,2)}$ \\ J. C. da S. Lacava ${ }^{(2)}$ \\ David Fernandes ${ }^{(2)}$ \\ ${ }^{(1)}$ Divisão de Processamento de Imagens, Instituto Nacional de Pesquisas Espaciais \\ Avenida dos Astronautas, 1758, CEP 12227-010, São José dos Campos-SP, Brazil \\ sidnei@dpi.inpe.br \\ ${ }^{(2)}$ Laboratório de Antenas e Propagação, Instituto Tecnológico de Aeronáutica \\ Praça Mal. Eduardo Gomes, 50, CEP 12228-900, São José dos Campos-SP, Brazil
}

\{lacava,david\}@ita.br

\begin{abstract}
The polarization plane rotation effects on SAR polarimetric attributes are analyzed. This analysis is carried out by simulating a polarimetric SAR image containing four regions with different electric characteristic. The simulation process is based on an electromagnetic scattering model. Employing a rotation model is generated a image set having 361 images, where each image has it polarization plane rotated by a different angle. From all images of the set are computed the covariance matrix and six polarimetric attributes of each image region and compared with their correspondent parameter in the image without rotation. From this evaluation it could be seen that the rotation angle influences differently all polarimetric attributes. The effects of rotation angle can lead to a misunderstanding of the target scattering mechanism.
\end{abstract}

Index Terms - polarization plane rotation, polarimetric SAR images, Faraday rotation

\section{INTRODUCTION}

The potential and usefulness of SAR (Synthetic Aperture Radar) data for mapping and monitoring land use and land cover classes have been proved in several researches over the past few decades [1]. The cloud cover over tropical regions, which is very common situation along of the year, can difficult (drastically affect) a systematic mapping using optical data since these kinds of data are strongly influenced by the atmospheric conditions. Therefore, the use of SAR data for monitoring tropical regions is highly indicated.

The orbital SAR data are recommended to map large areas, however depending of the operating frequency, the local time of data acquisition, the area's geographic location, solar activity, and the direction of the earth's magnetic field, among others factors, the SAR data can be strongly influenced by Faraday rotation. This rotation is a typical electromagnetic phenomenon, which is characterized by the rotation of the wave polarization plane, when this wave propagates through the ionosphere (a plasma medium). The strength of rotation will depend on the path of the electromagnetic wave, the physical properties of the ionosphere and the SAR operating frequency. According to [2] the Faraday rotation effects are more critical for SAR operating in low frequencies, as L- and P-bands.

The wave polarization plane rotation can leads to an erroneous interpretation of polarimetric SAR data, conducting to a misclassification of these kinds of data. In this context, the purpose of this work is to analyze the influence of wave polarization plane rotation on the some estimated polarimetric attributes.

\section{POLARIZATION PLANE ROTATION}

An electromagnetic wave is linearly polarized when its electric field always oscillates in the same plane. However when it propagates through an electrically active media such as magnetized plasma or a chiral medium, its polarization plane can be rotated. In Fig. 1 is illustrated, in simplified way, the polarization plane rotation of a linearly polarized wave. The wave is propagating in vector $\boldsymbol{k}$ direction and is polarized in $u$ direction. The propagation through an electrically active medium result in a rotation $\Delta$ and the rotated wave is linearly polarized in $u-\Delta$ direction.
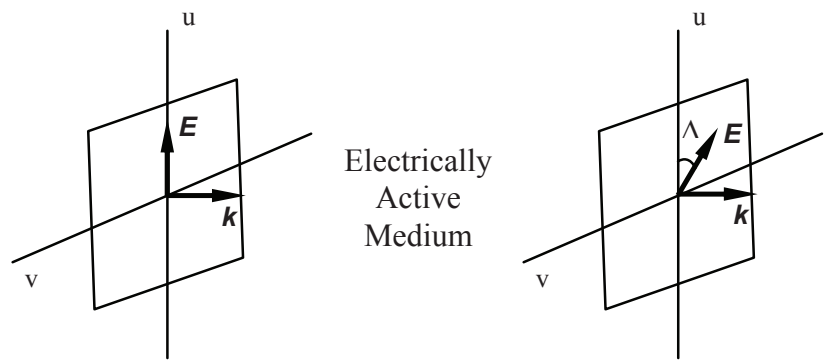

Fig. 1 - Plane polarization rotation.

In SAR imaging context the polarization plane rotation effect might be modeled through multiplying the target scattering matrix $[S]$ by unitary matrix of rotation $[R]$.

PROAP Project. 
Therefore, a polarimetric SAR operating in vertical and horizontal linear polarization will observe the target scattering matrix $[M]$ given by

$\left[\begin{array}{ll}M_{h h} & M_{v h} \\ M_{h v} & M_{v v}\end{array}\right]=\left[\begin{array}{cc}\cos \Delta_{1} & \operatorname{sen} \Delta_{1} \\ -\operatorname{sen} \Delta_{1} & \cos \Delta_{1}\end{array}\right]\left[\begin{array}{cc}S_{h h} & S_{v h} \\ S_{h v} & S_{v v}\end{array}\right]\left[\begin{array}{cc}\cos \Delta_{2} & \operatorname{sen} \Delta_{2} \\ -\operatorname{sen} \Delta_{2} & \cos \Delta_{2}\end{array}\right]$.

From equation (1) it can be seen that the $[S]$ matrix is multiplied by an $\left[R_{1}\right]$ and an $\left[R_{2}\right]$ unitary matrices. Each one multiplication is referred to the rotation in the paths sensor-target and target-sensor, respectively. Assuming that $\Delta_{1}=\Delta_{2}=\Delta$, i.e., the rotations are equal in both path and have identical sense, we obtain the same model proposed by Freeman e Saatchi [2]. This model was used to describe the Faraday rotation present in SAR data due to ionosphere effects, where the total rotation angle is considered for the round-trip range (downward and upward satellite links).

Analyzing the rotation model expressed in equation (1) under assumption that $\Delta_{1}=\Delta_{2}=\Delta$, it can be noted that if an imaged target is reciprocal (i.e., $S_{h v}=S_{v h}$ ) the measured scattering matrix $[M]$ will be reciprocal only if $\Delta= \pm m \pi / 2$, for $m=0,1,2 \ldots$. Using this model and varying the rotation angle $(\Delta)$ from $-180^{\circ}$ to $+180^{\circ}$ in step of $1^{\circ}$, it was generated 361 new sets of polarimetric SAR images. These new images (under the rotation effect) are generated by applying the equation (1) in the simulated SAR image, where the $[S]$ matrix is known pixel to pixel.

\section{SIMULATED IMAGE}

A $128 x 128$ pixels polarimetric image representing an acquisition of a monostatic SAR operating in L-band $(1.25 \mathrm{GHz})$ with grazing angle of $35^{\circ}$ was simulated using the methodology presented in [3]. The generated images, showed in Fig. 2, were based on an idealized image (a phantom image) containing four different regions. The local orientation of the elementary scatters (printed electric planar dipoles) was used as main electromagnetic characteristic to differentiate the regions. In the phantom red region (region $\mathrm{D}$ ) the dipoles were oriented totally random (TR) and in the magenta (region A), green (region C) and blue (region $\mathrm{B}$ ) regions the dipole have a local preferential orientation of $10^{\circ}, 20^{\circ}$ and $30^{\circ}$, respectively. This orientation is referred to the azimuth-axis (az) and the origin of the system coordinate is located at image center. The spatial resolution and pixel spacing

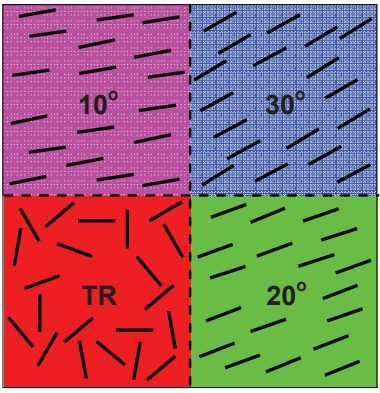

(a)

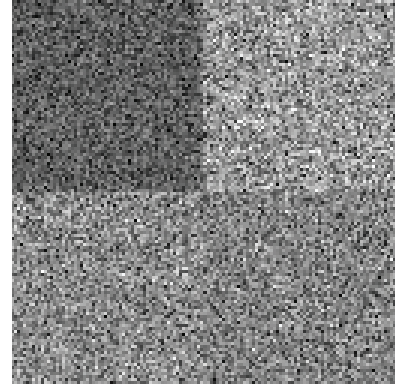

(b)

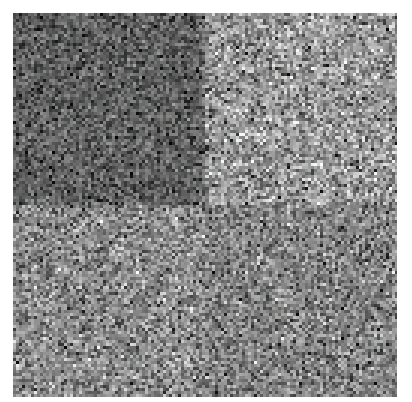

(c)

were fixed, respectively, at $3.0 \mathrm{~m}$ and $2.8 \mathrm{~m}$, both in range and azimuth directions. Dipoles sizing $50 \times 1 \mathrm{~mm}$ were used to represent the elementary scatters.

\section{POLARIMETRIC ATTRIBUTES ANALYSIS}

The data analysis was firstly carried out by comparing the covariance matrix for each image region with and without polarization plane rotation. The Manhattan, the Euclidean, the Bartlett and the revised Wishart distances between covariance matrices [4] were used in this comparison. If $\Sigma_{x}$ and $\Sigma_{y}$ are $p x p$ different covariance matrices, then the four distances are defined as:

1. Manhattan Distance $\left(\ell_{1}\right.$ metric)

$d_{1}\left(\Sigma_{x}, \Sigma_{y}\right)=\sum_{i \leq j}\left|\operatorname{Re}\left\{\Sigma_{x}-\Sigma_{y}\right\}_{i, j}\right|+\sum_{i \leq j}\left|\operatorname{dm}\left\{\Sigma_{x}-\Sigma_{y}\right\}_{i, j}\right|$

2. Euclidean Distance $\left(\ell_{2}\right.$ metric $)$

$d_{2}\left(\Sigma_{x}, \Sigma_{y}\right)=\sqrt{\sum_{i \leq j}\left|\left[\Sigma_{x}\right]_{i, j}-\left[\Sigma_{y}\right]_{i, j}\right|^{2}}$

3. Bartlett distance (Dissimilarity measure)

$d_{b}\left(\Sigma_{x}, \Sigma_{y}\right)=2 \ln \left(\frac{\left|\Sigma_{x+y}\right|}{\sqrt{\left|\Sigma_{x}\right|\left|\Sigma_{y}\right|}}\right)$

4. Revised Wishart Distance

$d_{w}\left(\Sigma_{x}, \Sigma_{y}\right)=\ln \left(\frac{\left|\Sigma_{x}\right|}{\left|\Sigma_{y}\right|}\right)+\operatorname{tr}\left(\Sigma_{x}^{-1} \Sigma_{y}\right)-p$

where $[A]_{i j}=a_{i j}$ is the indicial notation for the $(i, j)$ th element of matrix $A$, and $\left|[A]_{i j}\right|$ is the absolute value of $(i, j)$ th element of matrix $A$.

These four covariance distances were normalized by their respective maximum values, i.e., $d_{i n}=d_{i} / \operatorname{Max}\left\{d_{i}\right\}$, where $i \in\{1,2, b, w\}$ represents each distance and $n$ is referred to rotation angle. The normalization was used in order to unify the covariance matrices distances in only one distance measure. The unified distance measure is computed by the Euclidean distance $\left(d_{e}\right)$ between the point formed by the four normalized distances and the origin of the $\mathbb{R}^{4}$ space.

Fig. 2 - Simulated polarimetric SAR images: (a) Phantom, (b) HH, (c) HV and (d) VV.

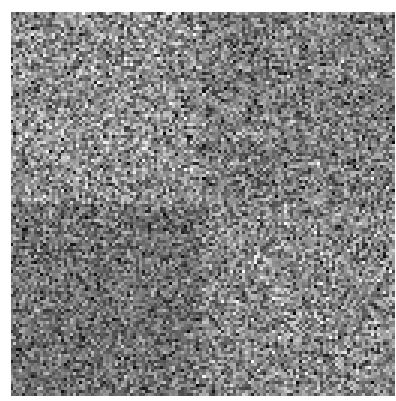

(d) 
A graphic showing the variation of $d_{e}$ in terms of the rotation angle $(\Delta)$ for each image region is depicted in Fig. 3. From this graphic it can be noticed the influence of rotation angle in the covariance matrix of each image region. It can also be noted that the effects of $\Delta$ angle on covariance matrices of $\mathrm{A}, \mathrm{B}$ and $\mathrm{C}$ regions are similar. For the rotation angle equal to $\pm 135^{\circ}$ and $\pm 45^{\circ} d_{e}$ reaches infinity value (square points in Fig. 3), due to Bartlett and Wishart distances which are infinity. The $\Delta$ angle modifies the covariance matrix of each region. This modification can lead to an erroneous interpretation of the target scattering mechanism. Therefore, for an orbital mapping using SAR data acquired with long wavelength it is very important to know ionosphere conditions.

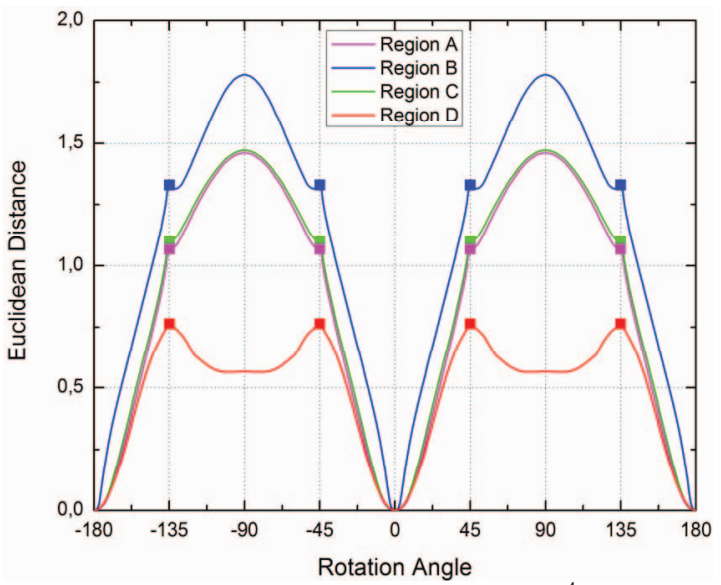

Fig. 3 - Euclidean distance variation in $\mathbb{R}^{4}$ space.

The effect of rotation angle in others six polarimetric attributes was also analyzed. These attributes include the $\alpha$ angle (derived from standard Cloude-Pottier decomposition [5]), the Co-Polarized Ratio (CPR), the Co-Polarized Phase Difference (CPPD), the Complex Co-Polarized Correlation Coefficient $(\rho)$ and two measures used to estimate moisture content: the polarization descriptor ratio (PDR), proposed in [6] and the polarization descriptor squared (PDS), proposed in [7]. These polarimetric attributes are defined as

\section{Alpha Angle}

$\alpha=\cos ^{-1}\left\{\frac{\left|S_{h h}+S_{v v}\right|}{\sqrt{\left|S_{h h}+S_{v v}\right|^{2}+\left|S_{h h}-S_{v v}\right|^{2}+4\left|S_{h v}\right|^{2}}}\right\}$

\section{Co-Polarized Ratio (CPR)}

$\mathrm{CPR}=\frac{\left\langle\left|S_{v v}\right|^{2}\right\rangle}{\left\langle\left|S_{h h}\right|^{2}\right\rangle}$
3. Co-Polarized Phase Difference $(C P P D)$

$\mathrm{CPPD}=\left\langle\operatorname{tg}^{-1}\left(\frac{\operatorname{Im}\left\{S_{h h}\right\}}{\operatorname{Re}\left\{S_{h h}\right\}}\right)-\operatorname{tg}^{-1}\left(\frac{\operatorname{Im}\left\{S_{v v}\right\}}{\operatorname{Re}\left\{S_{v v}\right\}}\right)\right\rangle$

4. Complex Co-Polarized Correlation Coefficient

$\rho_{h h-v v}=\left\langle\frac{S_{h h^{2}} S_{v v}^{*}}{\sqrt{\left\langle\left. S_{h h}\right|^{2}\right\rangle\left\langle\left. S_{v v}\right|^{2}\right\rangle}}\right\rangle$

5. Polarization Descriptor Ratio $(P D R)$

$\operatorname{PDR}=\frac{\left\langle\left|S_{v v}\right|^{2}\right\rangle-\left\langle\left|S_{h h}\right|^{2}\right\rangle}{\left\langle\left|S_{v v}\right|^{2}\right\rangle+\left\langle\left|S_{h h}\right|^{2}\right\rangle}$

6. Polarization Descriptor Squared (PDS)

$\operatorname{PDS}=\sqrt{\left\langle\left|S_{v v}\right|^{2}\right\rangle\left\langle\left|S_{h h}\right|^{2}\right\rangle}$

An image based on each polarimetric attribute was generated for each rotation angle. The difference between a rotated polarimetric attribute band and the polarimetric attribute band without rotation effect was calculated by the normalized root mean squared error (NRMSE). The NRMSE was computed taking into account the total number of pixels of each image region. It was verified that all polarimetric attributes were differently influenced by the rotation angle. As examples, are shown in Figs. 4 to 6 the variation of NMRSE versus $\Delta$ angle for CPPD, PDR and PDS attributes, respectively. The $\alpha$-angle attribute variation in terms of $\Delta$ angle is depicted in Fig. 7. From this figure it can be noted the great influence of rotation angle on the scattering mechanism, where its interpretability can be seriously modified.

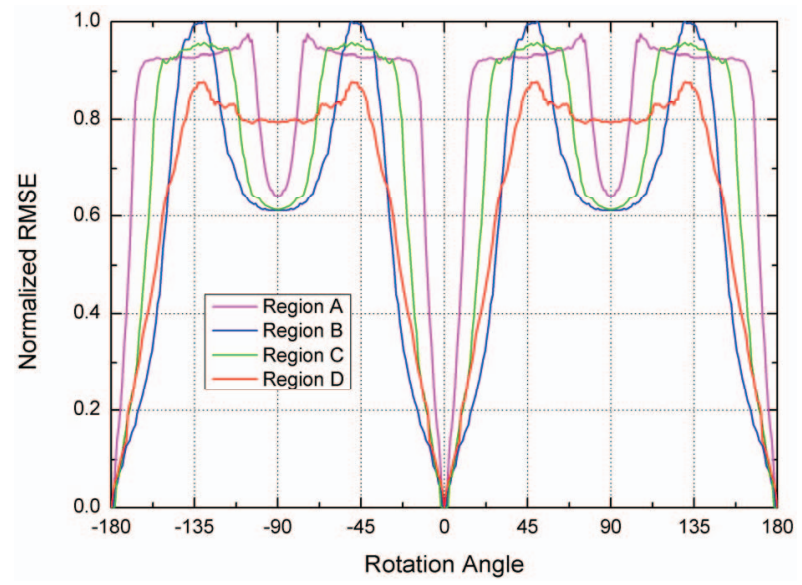

Fig. 4 - NRMSE for CPPD attribute. 


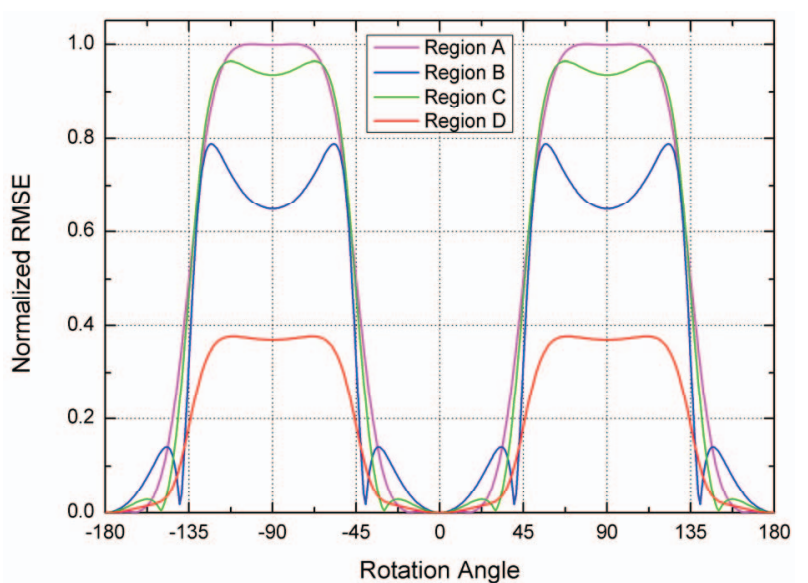

Fig. 5 - NRMSE for PDR attribute.

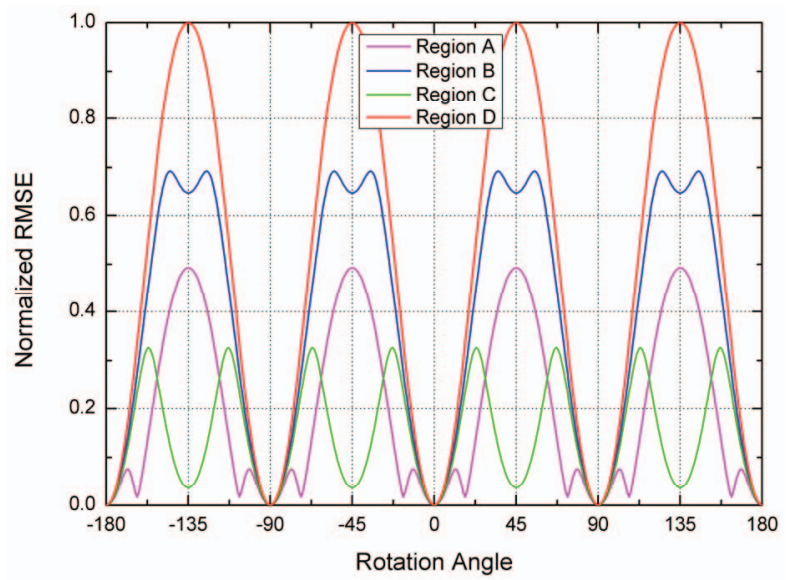

Fig. 6 - NRMSE for PDS attribute.

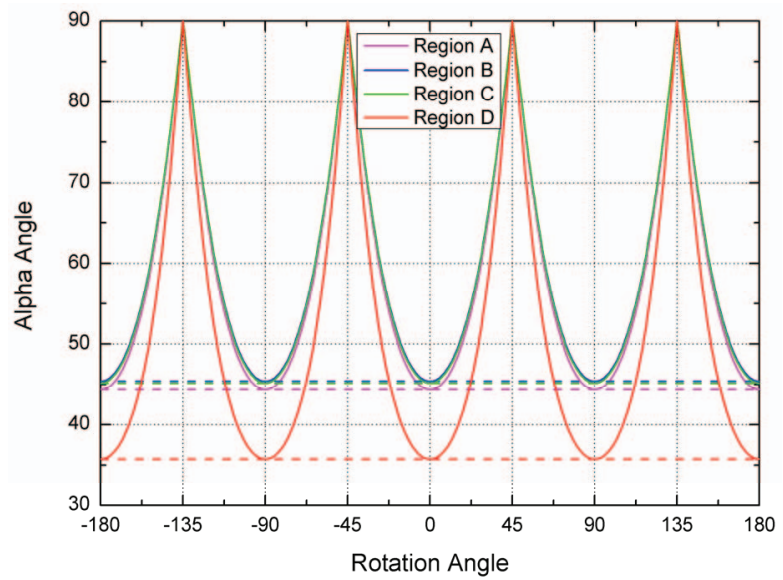

Fig. 7 - Variation of $\alpha$-angle attribute.

The influence of rotation angle on image segmentation process it was also verified. All 361 images were segmented using an interactive k-means algorithm based on the Wishart distance between covariance matrices. From this analysis it was observed that the segmentation algorithm, after five iterations, generates the same segmented image, i.e., the rotation angle does not affect the segmentation process. The segmented image is illustrated in Fig. 8, where all regions are very well discriminated. The segmented image presents only a small confusion in the regions' boundaries.

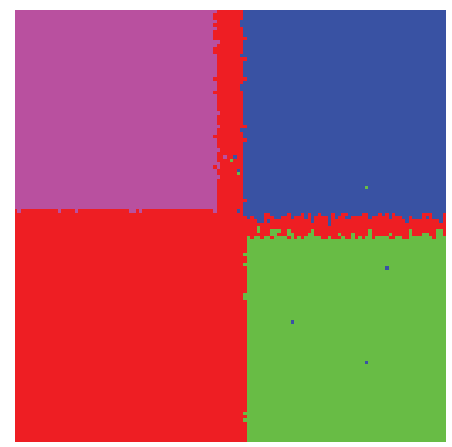

Fig. 8 - Segmented image.

\section{CONCLUSIONS}

From the results it was noted that the rotation angle affects differently all polarimetric attributes, including the covariance matrix, of each image region. Therefore, the orbital SAR data subject to Faraday rotation must be corrected before its use in mapping or monitoring land class and land use applications. The data correction is extremely important if a temporal series of SAR images is used for these applications.

\section{REFERENCES}

[1] C. C. Freitas, L. S. Soler, S. J. S. Sant'Anna, L. V. Dutra, J. R. Santos, J. C. Mura, and A. H. Correia, "Land use and land cover mapping in Brazilian Amazonia using polarimetric airborne P-band radar data," IEEE Transactions Geoscience and Remote Sensing, v. 46, n. 10, pp. 2956-2970, 2008.

[2] A. Freeman and S. S. Saatchi, "On the detection of Faraday rotation in linearly polarized L-Band SAR backscatter signatures," IEEE Transactions Geoscience and Remote Sensing, v. 42, n. 8, pp. 1607-1616, 2004.

[3] S. J. S. Sant'Anna, J. C. S. Lacava, and D. Fernandes, From Maxwell's equations to polarimetric SAR images: a simulation approach. Sensors, v. 8, n. 11, pp. 7380-7409, 2008.

[4] P. R. Kersten, J. S. Lee, and T. L. Ainsworth, "Unsupervised classification of polarimetric synthetic aperture radar images using fuzzy clustering and EM clustering," IEEE Transactions Geoscience and Remote Sensing, v. 43, n. 3, pp. 519-527, 2005.

[5] S. R. Cloude and E. Pottier, "An entropy based classification scheme for land applications of polarimetric SAR," IEEE Transactions Geoscience and Remote Sensing, v. 35, n. 1, pp. 68-78, 1997.

[6] D. Singh and V. Dubey, "Microwave bistatic polarization measurements for retrieval of soil moisture using an incident angle approach," J. Geophys. Eng., n. 4, pp. 75-82, 2007.

[7] T. Tadono, M. Qong, H. Wakabayashi, M. Shimada, T. Kobayashi, and J. Shi, "Preliminary studies for estimating surface soil moisture and roughness based on a simultaneous experiment with CRL/NASDA airbone SAR (PI-SAR)," In: Asian Conference on Remote Sensing 1999. 